\title{
End-to-End Optimized Nonlinear Fourier Transform-Based Coherent Communications
}

\author{
Gaiarin, Simone; Jones, Rasmus Thomas; Da Ros, Francesco; Zibar, Darko
}

Published in:

Optical Society of America

Link to article, DOI:

10.1364/CLEO_SI.2020.SF2L.4

Publication date:

2020

Document Version

Peer reviewed version

Link back to DTU Orbit

Citation (APA):

Gaiarin, S., Jones, R. T., Da Ros, F., \& Zibar, D. (2020). End-to-End Optimized Nonlinear Fourier TransformBased Coherent Communications. In Optical Society of America [SF2L.4] IEEE.

https://doi.org/10.1364/CLEO SI.2020.SF2L.4

\section{General rights}

Copyright and moral rights for the publications made accessible in the public portal are retained by the authors and/or other copyright owners and it is a condition of accessing publications that users recognise and abide by the legal requirements associated with these rights.

- Users may download and print one copy of any publication from the public portal for the purpose of private study or research.

- You may not further distribute the material or use it for any profit-making activity or commercial gain

- You may freely distribute the URL identifying the publication in the public portal

If you believe that this document breaches copyright please contact us providing details, and we will remove access to the work immediately and investigate your claim 


\title{
End-to-end optimized nonlinear Fourier transform-based coherent communications
}

\author{
Simone Gaiarin, Rasmus T. Jones, Francesco Da Ros, and Darko Zibar \\ DTU Fotonik, Technical University of Denmark, DTU, Kongens Lyngby, 2800, Denmark, simga@fotonik.dtu.dk
}

\begin{abstract}
We propose a jointly optimized nonlinear Fourier transform (NFT)-based transmitter and neural network-based receiver. More than two orders of magnitude improvement in bit error ratio is numerically reported for 5600-km transmission compared to a standard NFT system. (C) 2020 The Author(s)

OCIS codes: $060.1660,060.4510$.
\end{abstract}

\section{Introduction}

Nonlinear frequency division multiplexing (NFDM) is a modulation technique that analytically accounts for the Kerr nonlinearity of optical fibers, which is the main limiting factor to the performance of fiber optic communication systems. An NFDM system encodes data onto the so-called nonlinear spectrum of a signal using the nonlinear Fourier transform (NFT) [1]. The NFT is defined over an ideal fiber channel described by the lossless nonlinear Schrödinger equation (NLSE) [1] and over this channel it allows a distortion-free transmission.

However, realistic fiber channels include impairments such as losses and amplification noise, not accounted for by the NFT theory. To mitigate the negative impact of these additional effects on NFDM communication systems, several methods acting either at the transmitter or at the receiver have been proposed: equalizers [2], precoding methods [3], and receivers based on neural networks (NNs) [4].

In this paper we consider the full system optimization by jointly training an NFT-based transmitter and an NN-based receiver to maximize the overall NFDM transmission performance. Such end-to-end (E2E) optimization [5] is numerically applied to the full communication system including a transmission channel modelled through the split-step Fourier method (SSFM) [6]. The transmitter system parameters, nonlinear spectrum and power scaling, are jointly optimized together with the receiver NN. The analysis over a lossy and noisy channel shows that more than one and two orders of magnitude improvement in terms of bit error ratio (BER) can be achieved at $5600 \mathrm{~km}$ $(70 \times 80 \mathrm{~km}$ spans) through E2E optimization, when compared to a standard NFT receiver and an optimized NN receiver with unoptimized transmitter, respectively. It is shown that the improvement is preserved for at least up to \pm 10 spans from the optimized distance of 70 spans.

\section{Simulation setup}

The full simulation setup is shown in Fig. 1 and it is used for both the optimization and the performance evaluation of the system. At the transmitter we consider a nonlinear spectrum with two eigenvalues $\lambda_{i}$ and associated NFT coefficients $b\left(\lambda_{i}\right), i=1,2$. The modulation format over the $b\left(\lambda_{i}\right)$ is 4-phase-shift keying (PSK), i.e., $2 \mathrm{bit} / \mathrm{symbol} /$ eigenvalue at a symbol rate of $1 \mathrm{GBd}$. The transmitted single-polarization signal is generated by an inverse nonlinear Fourier transform (INFT) in order to assist the optimization procedure by restricting the solutions space to soliton-like pulse-shapes. These waveforms are matched to a channel, the ideal NLSE, similar to the one considered here. The channel consists of 80-km-long spans of single-mode fiber (SMF) $(D=17.5 \mathrm{ps} /(\mathrm{nm}$ $\mathrm{km}), \alpha=0.195 \mathrm{~dB} / \mathrm{km}$, and $\gamma=1.25(\mathrm{~W} \mathrm{~km})^{-1}$ ) and erbium-doped fiber amplifiers (EDFAs). At the receiver the real and imaginary parts of the samples of the time-domain waveform are fed to an $\mathrm{NN}$, which provides the most likely transmitted symbols on which the BER is finally estimated.

In detail, the transmitter operates as follows: each symbol $x_{j} \in\{1, \ldots 16\}$ of a random sequence is mapped onto a combination of the two $b\left(\lambda_{i}\right)$ taking values from the constellations $C_{i}=r_{i} \exp \left(j\left(k \frac{\pi}{2}+\theta_{i}\right)\right), k=0,1,2,3 ; i=1,2$

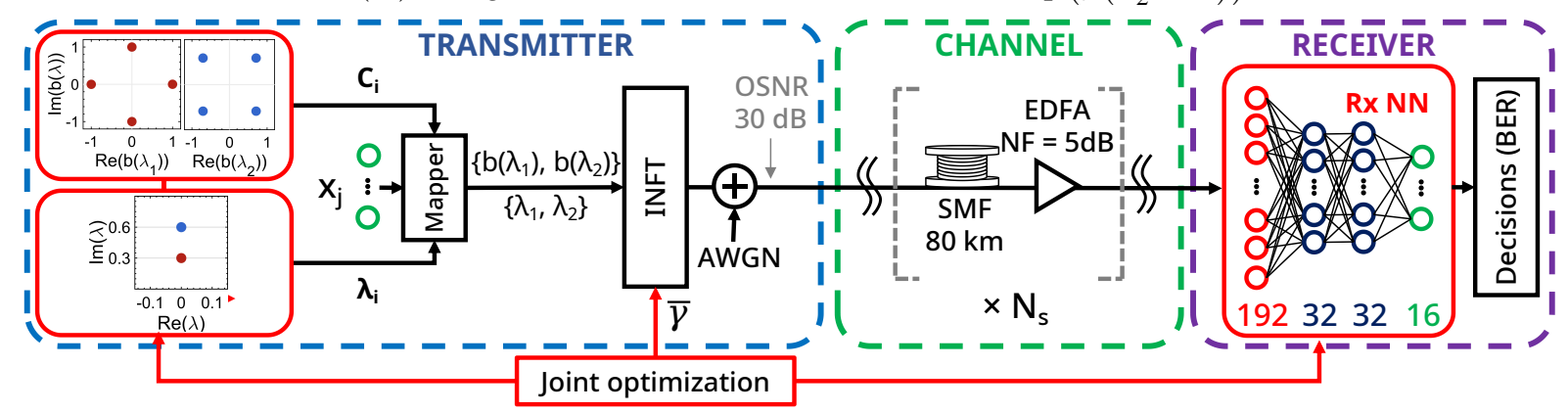

Fig. 1: Simulation setup used for the system E2E optimization and the BER performance evaluation. 


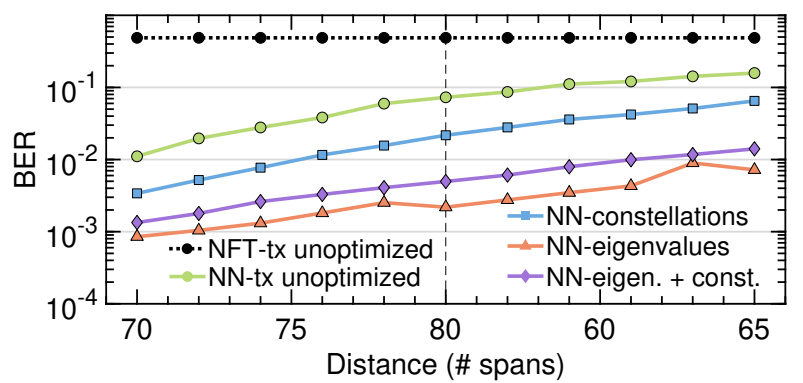

Fig. 2: BER as a function of the transmission distance for the NFT receiver with $\bar{\gamma}=\bar{\gamma}_{L P A}$ ('NFT') and for the NN receiver with $\bar{\gamma}=\bar{\gamma}_{E 2 E}($ 'NN'). The vertical dashed line marks the optimization distance.

with variable radii $r_{i}$ and phases $\theta_{i}$. Each NFDM symbol $\left\{\lambda_{i}, b\left(\lambda_{i}\right)\right\}$ is mapped by the INFT to a time-domain waveform (96 samples-per-symbol) using the Darboux transform (DT) with the time and power scaling parameters $T_{0}=47 \mathrm{ps}$ and $P_{n}=2 /\left(\bar{\gamma} T_{0}^{2}\right)$, respectively [1]. The parameter $\bar{\gamma}$ is an effective nonlinear coefficient.

The variables $\lambda_{i}, r_{i}$, and $\theta_{i}$ are either set to the reference values of $\lambda_{1}=0.3 j, \lambda_{2}=0.6 j, r_{1}=r_{2}=1, \theta_{1}=0$, $\theta_{2}=\pi / 4$ as in [4], or are provided by the E2E optimization performed in Tensorflow. The parameter $\bar{\gamma}$ controlling the transmitted signal power is optimized jointly with the other trainable variables $\left(\bar{\gamma}=\bar{\gamma}_{E 2 E}\right)$. The following combinations of trainable variables have been considered: $\left\{\left(\lambda_{i}, r_{i}, \theta_{i}\right)\right.$ fixed $\}$ ('tx unoptimized'), $\left\{\lambda_{i}\right.$ fixed; $\left(r_{i}, \theta_{i}\right)$ optimized $\}$ ('constellations'), $\left\{\left(r_{i}, \theta_{i}\right)\right.$ fixed; $\operatorname{Im}\left(\lambda_{i}\right)$ optimized $\}$ ('eigenvalues'), $\left\{\left(\operatorname{Im}\left(\lambda_{i}\right), r_{i}, \theta_{i}\right)\right.$ optimized $\}$ ('eigen. + const'). The E2E optimization was performed at a fixed distance of 70 spans $(5600 \mathrm{~km})$. The receiver NN is retrained on longer sequence of symbols sampled from the optimal nonlinear spectrum found by the E2E optimization to improve its performance. To compare the NN receiver results, a standard NFT receiver [7] is used as a benchmark. In this case $\bar{\gamma}$ is set to the lossless path-averaged (LPA) approximation value $\bar{\gamma}_{L P A}=\gamma\left(G_{a}-1\right) / \log \left(G_{a}\right)$ and $G_{a}=\exp \left(-\alpha L_{S}\right)$ [1] in both the INFT at the transmitter and the NFT at the receiver.

\section{Results and discussion}

Fig. 2 shows the BER performance (evaluated over $5 \times 10^{4}$ symbols) as a function of the transmission distance. In the reference case where an unoptimized constellation with an NFT receiver ('NFT-tx unoptimized') is considered, it is not possible to demodulate the data at any of the evaluated transmission distances (BER $\sim 0.5$ ). A demodulation is possible if the NFT receiver is replaced with the NN receiver and the factor controlling the transmitter power is set to the value found by the E2E optimization $\left(\bar{\gamma}=\bar{\gamma}_{E 2 E}\right)$. In this case, at the optimization distance of 70 spans the BER is $7.29 \times 10^{-2}$ ('NN-tx unoptimized'). Allowing a degree of freedom in the optimization of the nonlinear spectrum, ('NN-constellations' or 'NN-eigenvalues'), the BER is further reduced to $2.17 \times 10^{-2}$ and $2.19 \times 10^{-3}$ respectively. The system performance appears to be more sensitive to the optimization of the eigenvalues as $\operatorname{Im}\left(\lambda_{i}\right)$ is directly related to the noise variance $[3,7]$. Finally when the nonlinear spectrum is fully optimized ('NN-eigen+const') the BER is $4.97 \times 10^{-3}$. We observe that this case performs slightly worse than 'NN-eigenvalues', despite having more degrees of freedom. This is likely due to a local optima in the cost function from where the optimization procedure was not able to escape. This result further straighten our choice of using an NFT-based transmitter (theoretically ideal for a lossless/noiseless channel) to guide the E2E optimization. The curves also show that the performance gain is preserved for transmission distances between 60 and $80 \times 80 \mathrm{~km}$, even though the optimization was performed at 70 spans, showing the robustness of the optimization to the transmission distance.

\section{Conclusions}

An E2E optimization procedure for an NFDM transmission system employing an NN receiver and transmitting over a realistic fiber channel modelled by the SSFM has been proposed. The considered system outperforms both a system using an NFT receiver and one using an NN receiver with unoptimized transmitter providing an improvement of more than one and two orders of magnitude in terms of BER against them respectively. This demonstrates that the joint optimization of the transmitter with the receiver is critical to improve the system performance.

Acknowledgements: This work is supported by the European Research Council (ERC-CoG FRECOM project, 771878).

\section{References}

[1] A. Hasegawa and Y. Kodama, Solitons in Optical Communications, 7th ed. (Oxford University Press, USA, 1995).

[2] J. Koch, et al., "Equalization of Soliton Transmission Based on Nonlinear Fourier Transform using Neural Networks," in ECOC (2019).

[3] A. Span, et al., "Efficient Precoding Scheme for Dual-Polarization Multi-Soliton Spectral Amplitude Modulation," IEEE Trans. Comm. $1-13$ (2019).

[4] R.T. Jones, et al., 'Time-Domain Neural Network Receiver for Nonlinear Frequency Division Multiplexed Systems,” PTL 30, 1079--1082 (2018).

[5] O'Shea, et al., "An introduction to deep learning for the physical layer." IEEE Trans. on Cognitive Comm. and Networking, 563-575 (2017).

[6] Claude - End-to-End Learning of Optical Communication Systems, https://github.com/Rassibassi/claude

[7] S. Gaiarin, et al., "Dual polarization nonlinear Fourier transform-based optical communication system," Optica 5, 263--270 (2018). 